\title{
Ferromagnetic resonance, transverse bias initial inverse susceptibility and torque studies of magnetic properties of $\mathrm{CO}_{2} \mathrm{MnSi}$ thin films
}

\author{
M. Belmeguenai ${ }^{1}$, H. Tuzcuoglu ${ }^{1}$, D. Berling ${ }^{2}$, S.M. Chérif ${ }^{2}$, Y. Roussigné ${ }^{1}$, T. Devolder ${ }^{3}$ and K. Westerholt ${ }^{4}$ \\ ${ }^{1}$ LSPM (CNRS-UPR 3407) Université Paris 13, 99 avenue Jean-Baptiste Clément, 93430 Villetaneuse, France \\ ${ }^{2}$ IS2M (CNRS-LRC 7228) Université de Haute-Alsace, 15 Rue Jean Starcky BP2488, 68093 Mulhouse, France \\ ${ }^{3}$ IEF (CNRS UMR8622), Université Paris-Sud, 91405 Orsay, France \\ ${ }^{4}$ Experimentalphysik/Festkörperphysik, Ruhr-Universität Bochum, 44780 Bochum, Germany
}

\begin{abstract}
Magnetic properties of $\mathrm{Co}_{2} \mathrm{MnSi}$ thin films of $20 \mathrm{~nm}$ and $50 \mathrm{~nm}$ in thickness grown by radio frequency sputtering on a-plane sapphire substrates have been studied. X-ray diffraction (XRD) revealed that the cubic $<110>\mathrm{Co}_{2} \mathrm{MnSi}$ axis is normal to the substrate and that well defined preferential in-plane orientations are present. The static magnetic properties were studied at room temperature by conventional magneto-optical Kerr effect (MOKE), transverse bias initial inverse susceptibility and torque (TBIIST) MOKE. The dynamic magnetic properties were investigated by micro-strip ferromagnetic resonance (MS-FMR) at room temperature. The resonance and TBIIST measurements versus the direction of the in-plane applied magnetic field reveal that the in-plane anisotropy results from the superposition of a two-fold and a four-fold symmetry. The directions of the principal axes of the twofold anisotropy are sample dependent. The angular dependence of remanent normalized magnetizations and coercive fields, studied by MOKE are analyzed within the frame of a coherent rotation model. A good agreement is observed between the field anisotropy values obtained from MSFMR and from TBIIST data. Frequency and angular dependence of FMR linewidth has been studied. Apparent damping coefficient of 0.0112 has been measured for $50 \mathrm{~nm}$ thick sample.
\end{abstract}

\section{Introduction}

Cobalt-based full-Heusler with a general composition $\mathrm{Co}_{2} \mathrm{YZ}$ ( $\mathrm{Y}$ is element of the transition metal group, whereas the $\mathrm{Z}$ component comes from the group III-V elements) alloys are the most interesting class of Heusler materials for spintronic applications due to their predicted $100 \%$ spin-polarization and their high Curie temperature largely exceeding the room temperature. Moreover, tunnelling magnetoresistance (TMR) ratios overpassing $1000 \%$ have been reported [1] making such basedmaterial devices attractive for magnetic data storage applications.

$\mathrm{Co}_{2} \mathrm{MnSi}$ full Heusler, which has a Curie temperature of $985 \mathrm{~K}$, is one of the most studied full-Heusler alloys. It has been used in magnetic tunnel junctions with different barriers [2-4] where very high TMR ratios are obtained. Furthermore, recently, it was also reported the enhancement of the resistance change area product and large magnetoresistance ratio in current perpendicularto-plane giant magnetoresistance (CPP GMR) in epitaxially grown $\mathrm{Co}_{2} \mathrm{MnSi}$ based trilayers.

The key of a successful implementation of Heusler compounds into real devices, however, is a good understanding of their magnetic properties. The exchange stiffness constant $A_{e x}$ which expresses the trends of spins alignment in a magnetic material plays an important role in micromagnetic simulations and the study of dynamic phenomena. Moreover, the investigation of the magnetocrystalline anisotropies is a possible way to get insight of the spin-orbit interaction which would have a strong impact on the spin depolarization. Another technological interesting parameter is the Gilbert damping. Although low damping is essential for spin switching with low currents and spin torque oscillators, it enhances the spin-torque-induced magnetic noise in CPP GMR sensors.

The purpose of this paper is to use ferromagnetic resonance in microstrip line (MS-FMR) under an in-plane and out of plane applied magnetic field and combined transverse biased initial inverse susceptibility and torque (TBIIST) methods to study the magnetic properties of $\mathrm{Co}_{2} \mathrm{MnSi}$ thin films. The TBIIST method surely does not have the same reputation of FMR techniques but seems to be complementary to FMR especially for samples showing weak signals non detectable by FMR methods. We will show that TBIIST is a powerful static sensitive technique for the study of magnetic anisotropies and in 
particular provides an accurate value of the anisotropy field strength in the sample and a precise determination of the anisotropy axes.

\section{Sample and experimental set up}

$\mathrm{Co}_{2} \mathrm{MnSi}$ thin films (20 and $50 \mathrm{~nm}$ in thickness) were deposited on a-plane sapphire substrate using vanadium seed underlayer by UHV-magnetron rf-sputtering using pure Ar at a pressure of $5 \times 10^{-3}$ mbar as sputter gas and at $470^{\circ} \mathrm{C}$. After cooling them down to room temperature all the films were subsequently covered by a $5 \mathrm{~nm}$ thick gold layer protecting them against oxidation.

XRD measurements indicate that a $<110>$-type cubic axis is normal to the sample plane and the films behave as $\{110\}$ fiber textures. The $\mathrm{Co}_{2} \mathrm{MnSi}$ deduced cubic lattice constant (e.g.: $\mathrm{a}=5.658 \AA$ ) is in good agreement with the previously published value (5.654 $\AA$ ) [5].

The dynamic magnetic properties were studied using MS-FMR [6] setup piloted via a Labview program providing flexibility to real time control the magnetic field sweep direction, step and rate, real time data acquisition and visualization. This set-up which uses a vector network analyzer as generator allows measurements for both field and frequency sweeps. The resonance frequencies and fields are obtained from a fit assuming a lorentzian derivative shape of the recorded spectra. The obtained magnetic anisotropy parameters are compared to those determined from transverse biased initial inverse susceptibility and torque methods (TBIIST) [7]. In this technique, two magnetic fields $H_{L}$ and $H_{B}$ are applied in the plane of the film along directions defined by $\varphi_{H}$ and $\varphi_{H}+(\dot{2} 2)$, respectively, and the magnetization component $m_{L}$ is measured versus $H_{L}$ with a conventional magneto-optical Kerr experiment in longitudinal geometry. From the measured hysteresis loops, the initial inverse susceptibility $\left(\chi^{-1}\right)$ and the field offset $(\delta H)$ which are related to the second and first angle-derivative of the magnetic anisotropy, respectively, are obtained. Fourier analysis then easily resolves various contributions of different symmetries to the magnetic anisotropy and gives precise values for anisotropy fields and their easy axes.

\section{Magnetic properties}

\subsection{Static properties}

For the 20 and $50 \mathrm{~nm}$ films, MOKE hysteresis loops were measured versus external magnetic field orientations with respect to the c-axis of the substrate. The corresponding angular dependence of coercive fields $\left(H_{c}\right)$ and normalized remanent magnetizations $\left(M_{r} / M_{s}\right)$ are represented on figure 1 for the $50 \mathrm{~nm}$ sample. The two samples have a similar angular dependence of $H_{\mathrm{C}}$ and $M_{r} / M_{s}$ although the $H_{\mathrm{C}}$ magnitude and the easy axes directions are different from each other. Apparently, the angular dependences agree with those of Stoner Wohlfarth (coherent rotation: CR) model. It is not straightforward to guess the anisotropy nature and deduce anisotropy fields from these measurements. Basing on the TBIIST measurements shown on figure 2, the anisotropy nature and fields have been deduced. As in [6], the experimental data of $M_{r} / M_{s}$ and $H_{\mathrm{C}}$ have then been fitted considering a magnetic energy density which, in addition to Zeeman, demagnetizing and exchange terms, is characterized by the following anisotropy contribution:

$$
\begin{aligned}
& E_{\text {anis }}=-\frac{1}{2}\left[1+\cos 2\left(\varphi_{M}-\varphi_{u}\right)\right] K_{u} \sin _{M}^{2}+ \\
& K_{\perp} \sin ^{2}{ }_{M}-\frac{1}{8}\left[3+\cos 4\left(\varphi_{M}-\varphi_{4}\right)\right] K_{4} \sin ^{4}{ }_{M}
\end{aligned}
$$

In the above expression, $\theta_{M}$ and $\varphi_{M}$ respectively represent the out-of-plane and the in-plane (referring to the c-axis of the substrate) angles defining the direction of the magnetization $\boldsymbol{M}_{\boldsymbol{s}} ; \varphi_{u}$ and $\varphi_{4}$ stand for the angles of the easy uniaxial and of the easy fourfold anisotropies (represented by their anisotropy constants $K_{u}$ and $K_{4}$ respectively) axes, respectively, with this c-axis. $K_{\perp}$ is the perpendicular uniaxial anisotropy.

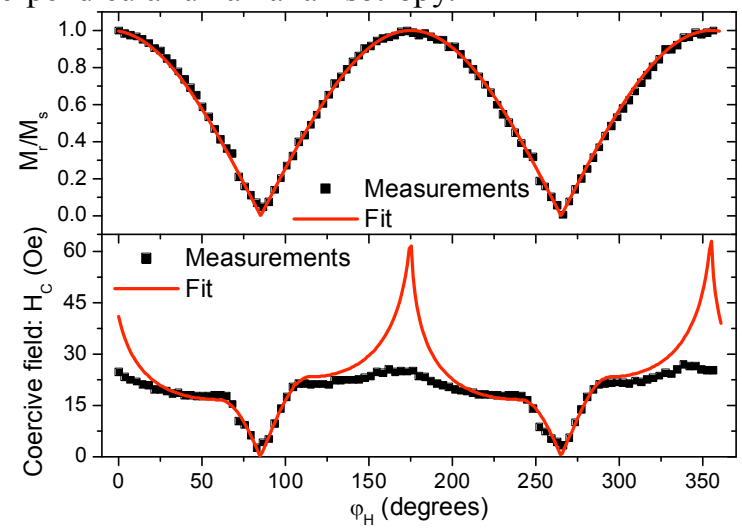

Fig. 1. (color online) Normalized remanent magnetization $M_{r} / M_{s}$ and coercive field $H_{C}$ of the $50 \mathrm{~nm} \mathrm{Co}_{2} \mathrm{MnSi}$ film.

Despite of the perfect agreement for $M_{r} / M_{s}$, a significant discrepancy for $H_{C}$ is shown around the easy axis direction between the CR model and measurements. In fact, the coercive fields found in the magnetization loops around the easy axis are smaller than the one obtained from CR model since that they are usually determined by domain nucleation and sample imperfection. The best parameters used for the fit of TBIIST and MOKE measurements are summarized in table 1. The samples present a fourfold anisotropy superimposed to uniaxial anisotropy. In both films the caxis of the substrate coincides with a principal direction of the fourfold magnetic anisotropy: it defines a hard axis $\left(\varphi_{4}=45^{\circ}\right)$ for $20 \mathrm{~nm}$ in contrast to the $50 \mathrm{~nm}$ sample for which it defines an easy axis $\left(\varphi_{4}=0^{\circ}\right)$. The directions of the principal axes of the twofold anisotropy are sample dependent. The observed variations of the in-plane magnetic anisotropy are not clearly related either to the thickness or to the crystallographic texture (which does not significantly change). During the preparation of the films uncontrolled parameters presumably induce different stress conditions giving rise to changes in the magnetic anisotropy. 


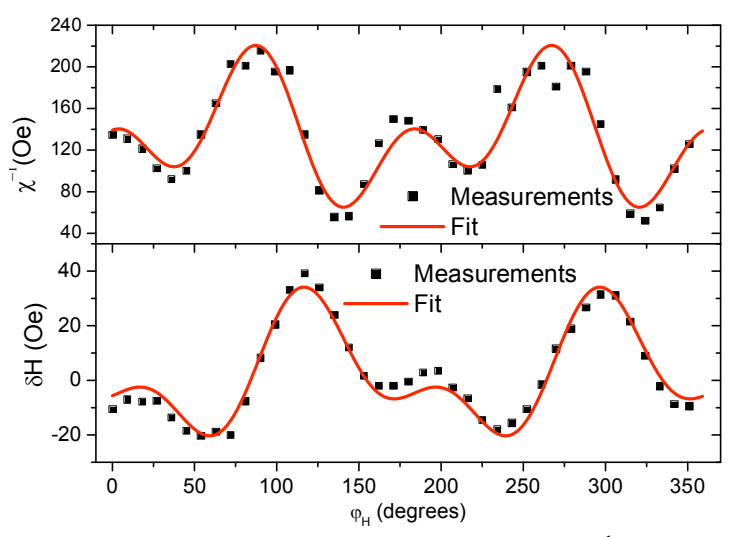

Fig. 2. (color online) Inverse susceptibility $\left(\chi^{-1}\right)$ and the field offset $(\delta H)$ of $50 \mathrm{~nm} \mathrm{Co} \mathrm{CnSi}_{2} \mathrm{Milm}$. The measurements were obtained using transverse static bias field $\mathrm{H}_{\mathrm{B}}=125$ Oe.

\subsection{Dynamic properties}

The experimental results are interpreted by a model that takes into account the most relevant energy contributions, such as Zeeman, uniaxial, and surface anisotropies (equation (1). The resonance condition is given by:

$$
F_{r}^{2}=\left(\frac{\gamma}{2 \pi}\right)^{2} \frac{1}{M_{s}^{2} \sin ^{2} \theta_{M}}\left[\frac{\partial^{2} E}{\partial \theta_{M}^{2}} \frac{\partial^{2} E}{\partial \varphi_{M}^{2}}-\left(\frac{\partial^{2} E}{\partial \theta_{M} \partial \varphi_{M}}\right)^{2}\right]
$$

The partial derivatives are evaluated at the equilibrium angles of the magnetization. We define $M_{\text {eff }}=M_{s}-\frac{K_{\perp}}{2 \pi M_{s}}, H_{u}=\frac{2 K_{u}}{M_{s}}$ and $H_{4}=\frac{4 K_{4}}{M_{s}}$ as the effective magnetization, the in-plane uniaxial and the fourfold anisotropy fields respectively.

The MS-FMR technique, in perpendicular configuration, allows for deriving the values of $g$ and of $4 \pi M_{\text {eff }}$ from the variation of the resonance frequency versus the magnitude of the applied field. The MS-FMR field-dependences of the resonance frequencies of the uniform and of the perpendicular standing spin wave (PSSW: detectable only for $50 \mathrm{~nm}$ thick film) modes are shown on figure 3 for both samples. The frequencies vary linearly with $H$. The derived value of $g$, using the model presented in [6] is independent of the sample $g=2.04$. The effective demagnetizing field $4 \pi M_{\text {eff }}$ slightly increases versus the sample thickness but remains close to the saturation magnetization $(12200 \mathrm{Oe})$ given by Hamrle [8]: it is reported in Table 1. The best fits for the observed PSSW are obtained using $A_{e x}=2.87 \mu \mathrm{erg} / \mathrm{cm}$. The in-plane angular dependence of the resonance frequency (sweep frequency), resonance field (sweep field at different frequencies) and peak to peak field FMR linewidth $\left(\Delta H^{P P}\right)$ have been measured for each sample. The field angle with the c-axis has been varied from $0^{\circ}$ to $360^{\circ}$ by step of $10^{\circ} . \Delta H^{P P}$ has been taken as the separation in field between the extrema of the FMR derivative curves. The typical obtained results are represented on figure 4 for the $50 \mathrm{~nm}$ thick sample and compared to the best fit using the above mentioned model.

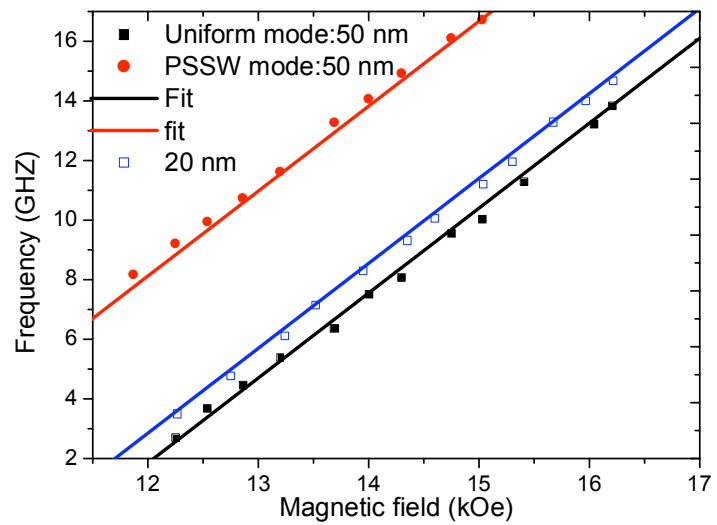

Fig. 3. (color online) Uniform precession and perpendicular standing spin waves (PSSW) modes frequencies versus the perpendicular applied magnetic field for 20 and $50 \mathrm{~nm}$ thick $\mathrm{Co}_{2} \mathrm{MnSi}$ films. The solid lines refer to the fit suing the above mentioned model.

For all the samples, the obtained values of the magnetic parameters corresponding to the best fits reported in Table 1 are in good agreement with those obtained from the fit of the TBIIST measurements. The resonance field and frequency behavior is governed by a superposition of fourfold and uniaxial anisotropies having the c-axis of the substrate as an easy or hard axis. The difference and change in the fourfold anisotropy axes between the 20 and $50 \mathrm{~nm}$ thick samples are maybe due to the different local stress, sample process, growth condition and to the in-plane complex film structure. It was not possible to us to derive a quantitative understanding of the anisotropies resulting from the real film structure.

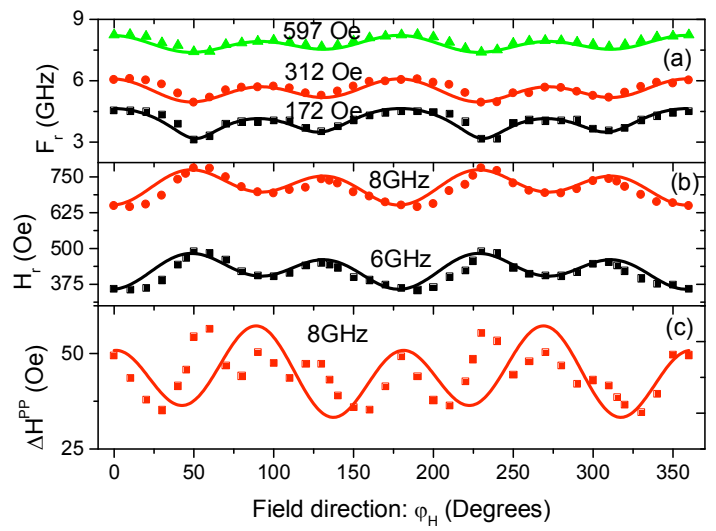

Fig. 4. (color online) Angular dependence of the resonance frequency $\left(F_{r}\right)$, resonance field $\left(H_{r}\right)$ and peak to peak field FMR linewidth $\left(\Delta H^{P P}\right)$ for $50 \mathrm{~nm}$ thick $\mathrm{Co}_{2} \mathrm{MnSi}$ film. The solid lines refer to the fit suing the above mentioned model.

Figure $4 \mathrm{c}$ shows clear variations of $\Delta H^{P P}$ versus the applied field direction for $50 \mathrm{~nm}$ thick film. The frequency dependence of this linewidth for an applied field along the easy axis is represented on figure 5.

Table 1. Magnetic parameters obtained from the best fits to our experimental results using MS-FMR and TBIIST techniques. $\varphi_{u}$ and $\varphi_{4}$ are the angles of the in-plane uniaxial and of the fourfold anisotropy easy axes, respectively. $\alpha_{a p p}$ is the apparent damping. 
The experimental results were analyzed considering intrinsic and extrinsic mechanisms. The intrinsic contribution, which in our case has no angular dependence due the fact that the applied fields and magnetization are parallel, traduces the apparent Gilbert damping. In order to describe the extrinsic contribution in the FMR linewidth, the most relevant mechanisms, such as two magnon scattering, mosaicity and inhomogeneities, have been taken into account. However, the large angular variation of $\Delta H^{P P}$ in our case cannot only be caused by mosaicity, which traduces variations in the internal fields, thickness, or orientation of crystallites within the sample. Dispersion of $1^{\circ}$ of anisotropy axis, which in our opinion remains far from reality and obtained values in practice, gives $\Delta H^{P P}$ variations of $4 \mathrm{Oe}$ at $8 \mathrm{GHz}$. Although two magnon scattering can gives rise to high variation of the angular dependence of linewidth in our case, it cannot perfectly reproduce its shape in our case (Fig. 4c). It is therefore more probably that other mechanisms like inhomogeneities, are responsible of such behavior of $\Delta H^{P P}$. Indeed, for some field orientations (around hard axis), the peak shows a complex shape suggesting that the measured FMR spectrum is a superposition of more than one peak maybe due to the complex in-plane structure of films revealed by XRD (not shown here).

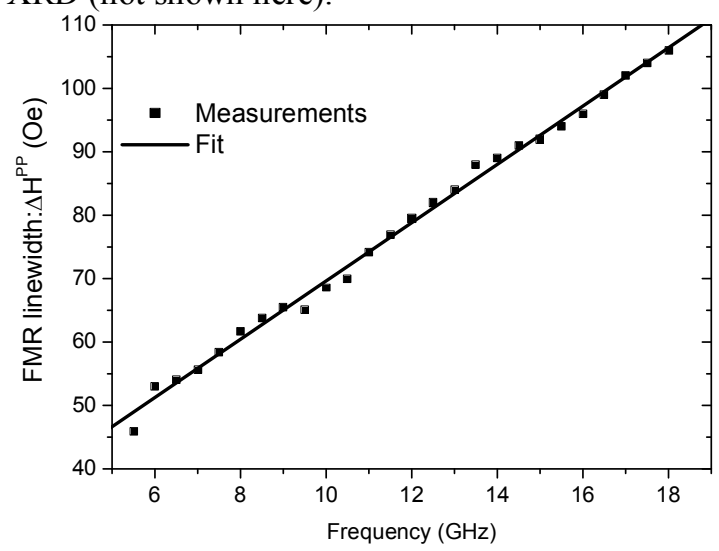

Fig. 5. Frequency dependence of the peak to peak field FMR linewidth $\left(\Delta H^{P P}\right)$ for $50 \mathrm{~nm}$ thick $\mathrm{Co}_{2} \mathrm{MnSi}$ film. The solid lines refer to the fit suing equation (3).

We thus tried to fit the variation of linewidth versus frequency using the expression (3):

$\Delta H^{P P}=\frac{2}{\sqrt{3}} \frac{2 \pi}{\gamma} \alpha_{\text {app }} f+\Delta H^{P P}(0)$

$\Delta H^{P P}(0)$ describes linewidth arising from inhomogeneous broadening.

The obtained value of apparent damping $\alpha_{a p p}=0.0112$ for the $50 \mathrm{~nm}$ thick film (see table) lay in the range of the observed ones [9]. It is also in good agreement with that of the $20 \mathrm{~nm}$ thick film (see table) measured with an applied field perpendicular to the sample plane where two magnon scattering contribution vanishes.

Due to the high values of $4 M_{\text {eff }}$ for our samples, the frequency variation of $\Delta H^{P P}$ does not allow for

\section{$42 \pm 6.5 \quad 88 \pm 5 \quad 93 \pm 7.5 \quad-13 \pm 2 \quad-12 \pm 1.60 \pm 2 \quad 0 \pm 0.85$}

evidencing the existence of the two magnon contribution in contrast to its in-plane angular dependence.

\section{Conclusion}

$\mathrm{Co}_{2} \mathrm{MnSi}$ films of $20 \mathrm{~nm}$ and $50 \mathrm{~nm}$ in thicknesses were prepared by sputtering on a a-plane sapphire substrate. They show practically identical crystallographic textures, as revealed by our X-rays diffraction studies, with a cubic [110] axis normal to the film-plane and with a well defined manifold of in-plane orientations referring to the c-axis of the substrate. The micro-strip ferromagnetic resonance (MS-FMR), transverse biased initial inverse susceptibility and torque (TBIIST) methods have been used to study their dynamic and static properties. The inplane anisotropy presents two contributions, showing a four-fold and a two-fold axial symmetry, respectively. A good agreement between parameters deduced from the fit of MS-FMR and TBIIST measurements has been obtained.

\section{References}

1. T. Ishikawa, H.-X. Liu, T. Taira, K.-I. Matsuda, T. Uemura, and M. Yamamoto, Appl. Phys. Lett. 95, 232512 (2009)

2. J. Schmalhorst, S. Kämmerer, M. Sacher, G. Reiss, A. Hütten, and A. Scholl, Phys. Rev. B 70, 024426 (2004)

3. K. Inomata, N. Ikeda, N. Tezuka, R. Goto, S. Sugimoto, M. Wojcik, and E. Jedryka, Sci. Technol. Adv. Mat. 9, 014101 (2008)

4. T. Ishikawa, S. Hakamata, K. Matsuda, T. Uemura, and M. Yamamoto, J. Appl. Phys. 103, 07A919 (2008)

5. P. J. Webster, J. Phys. Chem. Solids 32, 1221 (1971)

6. M. Belmeguenai, F. Zighem, Y. Roussigné, S.M. Chérif, P. Moch, K. Westerholt, G. Woltersdorf, and G. Bayreuther Phys. Rev. B 79, 024419 (2009)

7. D. Berling, S. Zabrocki, R. Stephan, G. Garreau, J.L. Bubendorff, A. Mehdaoui, D. Bolmont, P. Wetzel, C. Pirri and G. Gewinner, J. Magn. Magn. Mat. 297, 118 (2006)

8. J. Hamrle, O. Gaier, Seong-Gi Min, B. Hillebrands, Y. Sakuraba and Y. Ando, J. Phys. D 42, 084005 (2009)

9. R. Yilgin, M. Oogane, Y. Ando and T. Miyazaki, J. Magn. Magn. Mat. 310, 2223 (2007) 